\title{
A Virtual Museum Installation for Time Travel
}

\author{
Adeola Fabola ${ }^{1}$, Sarah Kennedy ${ }^{1}$, Alan Miller ${ }^{1}$, Iain Oliver ${ }^{1}$, John McCaffery $^{1}$, \\ Catherine Cassidy $^{1}$, Jo Clemens ${ }^{2}$, and Anna Vermehren ${ }^{2}$ \\ 1 School of Computer Science, Universitiy of St Andrews, United Kingdom \\ aef6, sk383, alan.miller, iao, jm726r, cc274@st-andrews .ac.uk \\ http://www.cs.st-andrews.ac.uk/ \\ 2 Timespan Museum \& Arts Centre, Helmsdale, United Kingdom \\ archivedevelopment, director@timespan.org.uk \\ http://www.timespan.org.uk/
}

\begin{abstract}
This work discusses the methodology for the design, development and deployment of a virtual $19^{\text {th }}$-century Fish Curing Yard as an immersive museum installation. The museum building now occupies the same space where the curing yard was over 100 years prior, hence the deployment of a virtual reconstruction of the curing yard in a game engine enables the museum visitors to explore the virtual world from equivalent vantage points in the real world. The project methodology achieves the goal of maximising user experience for visitors while minimising cost for the museum, and focus group evaluations of the system revealed the success of the interaction-free design with snackable content. A major implication of the findings is that museums can provide compelling and informative experiences that enable visitors to travel back in time with minimal interaction and relatively low cost systems.
\end{abstract}

Keywords: Virtual reality, virtual museum, immersion, 3D reconstruction

\section{Introduction}

A museum installation for exploring the past has been designed, implemented and evaluated in this work. The system features immersive, yet inexpensive technology which allows museum visitors to facilitate a comparison of the past and present from equivalent vantage points. Museum visitors can walk up to the installation in the foyer, pick up a virtual reality headset and look into the past to explore a $19^{\text {th }}$-century curing yard which occupied the space that the museum building occupies today.

The requirement to explore the past from fixed locations around the museum building has informed the decision to deploy the exhibit as high-fidelity photospheres ( $360^{\circ}$ panoramic images) instead of a resource-intensive 3D model powered by a game engine. Hence the use of immersive, yet inexpensive technology to facilitate a comparison of the past and present from equivalent vantage points in a museum setting is the major contribution of this work. Furthermore, an interaction-free design which provides snackable content to visitors thus enabling a group of people (a coach tour for example) to explore the past and share their experiences with one another, offers a contribution in the design of immersive virtual museum installations. An iterative methodology has been 


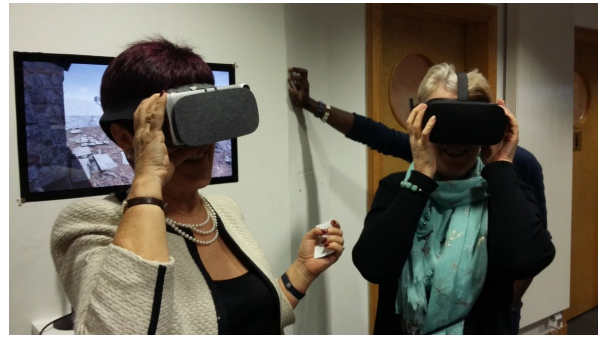

Fig. 1: Visitors with VR headsets

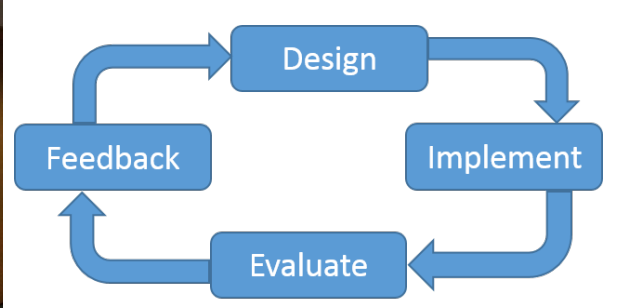

Fig. 2: Research Methodology

adopted for the design and implementation of the system, and evaluation has been conducted which demonstrates the success of the system for exploring the past as well as the acceptance of the interaction-free design. The findings of the evaluation also reveal insights into how visitors interact with virtual museum installations as well as the considerations that (staff and administrators of) museums face when managing such installations.

The remainder of the paper is organized as follows. Section 2 provides a background discussion on virtual museums and the partnership (and previous work) that led to the conception of the project. Section 3 discusses work done on the use of 3D technologies for museum installations and heritage visualisation. Section 4 provides an overview of the methodology adopted for the project, and Section 5 discusses the design and implementation of the system, highlighting the reconstruction process and the exhibit installation. The results of the system evaluation are provided in Section 6 and Section 7 concludes the work.

\section{Background - Virtual Musems and 3D Media}

The term "Virtual Museum" refers to either (1) the recreation of a physical museum, like-for-like which enables visitors to remotely simulate a virtual visit of the museum or (2) the creation of artefacts, items or experiences for users' consumption. This could be done remotely (on the Internet) or on-site (in a physical museum) 1. The second definition is of pertinence to this work, as it introduces the design, deployment and evaluation of an immersive museum installation aimed at providing an educational resource on local history.

Virtual museums have gained popularity in recent decades [2, and projects that leverage computer graphics to re-enact historical scenes date back the the early 1980s [3. Like recent interest in using 3D computer imagery and graphics to deliver engaging experiences to users, previous generations leveraged the techniques at their disposal in order to enhance immersive experiences [4]. The use of Panoramas (and similar film-based techniques such as Cineorama, Diorama, Georama and Stereorama) for cultural heritage applications date back to the $18^{\text {th }}$-century. These systems and their concomitant technologies were leveraged due to their ability to "involve" audiences and place them at the centre of the scene. However, due to content and cost limitations, the popularity of these technologies declined as people sought alternatives [5]. The use of panoramas as part of a general body of spherical and 3D media has seen a resurgence in recent years. This trend can be attributed (in part) to advances in photography, computer graphics, mobile and digital technologies. This, coupled with the desire 
to visualise the past and explore geographically-distant landscapes [5], provides new opportunities and facilitate the development of systems that enable us to bridge time (historic) and space (geographic) barriers.

Timespan museum and arts centre is a cultural organisation located in Helmsdale, a small town on the north-east coast of Scotland. Timespan previously collaborated with the Open Virtual Worlds (OVW) research group at the University of St Andrews to mark the $200^{\text {th }}$ anniversary of the Scottish Highland Clearances by producing a Clearances Trail App and an immersive CAEN Township installation [6. The Clearances Trail App is a mobile app (available on Android and $\mathrm{iOS}$ ) that enables users to take part in a guided tour of the Strath of Kildonan, which was central to the Highland Clearances in the $19^{\text {th }}$-century. The tour combines text, audio narratives, images and a location-aware map to educate users on the history of the Highland Clearances. The CAEN Township installation is an immersive exhibit which features a Microsoft Kinect and three large screens, situated in a "story-telling room", that enables visitors to visualise and explore a 3D model of CAEN, a pre-clearances settlement in the Scottish Highlands. Users can move around the model, enter longhouses, trigger informative pop-ups, listen to stories and watch animated movies, all of which are designed to educate the audience and provide them with a sense of the Highlands before the Clearances.

The building which Timespan now occupies was a fish curing yard around the late $19^{\text {th }}$ and early $20^{\text {th }}$-century. Fishing was a major source of income and livelihood for the inhabitants of $19^{\text {th }}$-century Helmsdale, hence the curing yard played an important role in the village's economy as it was a space where the herring and salmon caught by fishermen were gutted, cured and packaged for transportation. The ability to re-live and explore the curing yard as it stood in the $19^{\text {th }}$-century thus serves as a great resource for preserving and disseminating the heritage for the locals, and provides a medium for tourists to learn and engage with local history. This is the motivation of this work.

As part of Timespan's $30^{\text {th }}$ anniversary a project was conceived to celebrate local history. The aim of the project was to produce an immersive museum installation that would serve as an educational resource for the village locals as well as offer novel, interactive experiences to the museum visitors, some of whom are foreign travellers passing through the Highlands, and the ongoing collaboration with the OVW research group and the success of previous projects provided a good platform to execute the project.

\section{Related Work}

The use of 3D technologies to recreate and visualise history has been documented extensively in literature. The reconstruction of an important but now derelict cathedral as it stood in the $14^{\text {th }}$-century is documented in 7 . The work describes the reconstruction process including interdisciplinary research, building the landscape based on Ordinance Survey data, establishing the architecture, and embedding sound and scripted Non-Player Characters (NPC) such as canons and historical figures. The resulting model and associated content have been deployed in learning contexts such as schools (for primary and secondary education), festivals (for community engagement), and on the web 
(accessible remotely through a browser). A framework for building interactive virtual museum content and exhibitions is proposed in 8 . The system leverages a popular game engine in addition to web frameworks to provide a distributed service (based on data pulled from popular online repositories) that enables users to easily create, manage and share virtual exhibits which are not limited to a specific application domain, but rather support a broad range of applications. A system for visualising 3D models of Mediterranean sculptures, optimised for both small (mobile) and large (desktop) screens is presented in 9. The system is capable of streaming content over a network and displaying content at multiple resolution levels so as to improve performance and facilitate the inspection of the model in high levels of detail. In contrast to [9], which combines a mobile-based and desktop-based approach, 10, adopts a purely mobile-based approach to heritage visualisation. The system, which features cross-platform support (made possible by web technologies), enables users to visualise a 3D model of a mediaeval town with minimal resources. The impact of 3D technologies in the domain of cultural heritage is investigated in [11, and the findings affirm the case for using digital capture approaches such as photogrammetry and laser scanning to foster community engagement with cultural heritage, as well as the use of immersive technologies such as virtual reality headsets to provide compelling experiences thus increasing users' engagement with heritage content. These work demonstrate different ways in which 3D technologies can be used to recreate and visualise the past. The reconstruction process described in 7], the user-centric approach adopted in [8], the multi-platform approach adopted in [9] and [10, and the findings of [11] have been given due consideration in the actualisation of this project.

Furthermore, remote virtual museum visits in older adults result in a positive experience and high usability when an interaction-free design is adopted [12], such that users can consume content with little or no active interaction. This provides rationale for the interaction-free design adopted in this work. It is worth noting that the virtual museum presented in [12] facilitates a remote visit, contrary to the system designed in this work which allows visitors to explore the past on-site. Nonetheless, the design principles remain relevant because the primary objective is to present "snackable" content to visitors thus providing an informative and engaging experience in little time.

Technological advances, the proliferation of smart devices and 3D media, and the corresponding reduction in cost have contributed to the popularity of virtual museums in recent decades. These technologies enable the development of virtual exhibits that mitigate the risk of damage to artefacts and require little or no real estate [2]. The concept of the "Museum of Pure Form", proposed in [13, involves the use of virtual reality devices to provide immersive experiences to visitors. It overcomes a limitation of traditional museums (in which visitors cannot go close to or touch exhibits) by providing haptic feedback to simulate the sense of touch while interacting with digital replicas of the exhibits, usually coupled with stereoscopic visual display of the digital replica for immersion. In addition to bodily-immersive technologies, spatially-immersive technologies have also been deployed in museum contexts. The use of a panoramic stereo screen to present artwork to museum visitors is presented in [14, which documents a high degree of interaction with, and immersion in the virtual environment. In a similar vein, [6] reports on the recreation of a $19^{\text {th }}$-century township and its deployment in a 


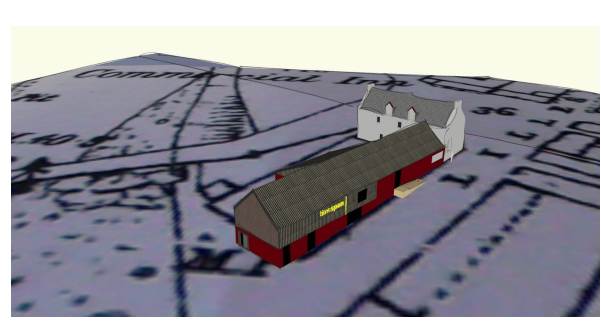

Fig. 3: Preliminary model in Sketchup

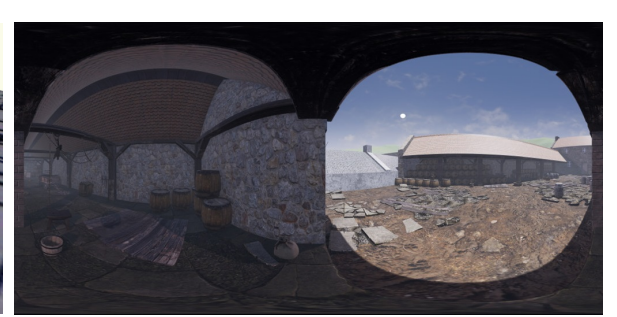

Fig. 4: Equirectangular image of the yard

museum, which enables visitors to explore the township through body gestures. The installation uses the Microsoft Kinect for motion detection and three large projection screens arranged as a semi-hexagon to produce a $150^{\circ}$ field of view. The common denominator of the aforementioned work - and what makes them relevant to this work - is their use of technologies in museum contexts, either to replicate museum exhibits which can be accessed off-site, or to create virtual environments which can be deployed on-site. This demonstrates the maturity of virtual museums and the versatility of immersive technologies; for instance, body-based devices are used in [13, while space-based devices are used in [6] and [14, all with the common goal of giving users a sense of presence in a virtual world. It is worth noting that the system proposed in this work uses neither haptic (as in [13]) nor spatially-immersive (as in [6] and [14]) technologies owing to cost and space constraints respectively, but rather features a head-mounted display and a screen (slotted into a false wall) to deliver an immersive experience in while minimising cost and space requirements.

In summary, computer graphics and 3D technologies have facilitated the recreation of historical scenes and artefacts, which have been used in the development of exhibits that offer interactive and immersive experiences to museum visitors. These developments have been leveraged in this work, with the contribution of enabling museum visitors to compare a recreated historical scene and its present day counterpart from an equivalent vantage point.

\section{Methodology}

The methodology for this work was one of a practice-based research. During an initial research phase, heritage experts at the museum consulted archival documents from the local and neighbouring museums. Two of the primary sources used for research include [15, which sets out the plan for the first curing yards in Helmsdale and 16, the annual reports of the British Fisheries Society - the industry regulator. Based on these sources, as well as maps and plans of the area, a preliminary 3D model was created using Unreal Engine 4 [17. This model then served as the basis for eliciting feedback from the heritage experts and locals, which provided further input for refining the model. An iterative designimplement-feedback cycle (see Fig 2 led to the development of a model that was deployed and evaluated with the visitors and heritage experts at the museum.

A prototype of the system was trailed at the Helmsdale Highland Games in the summer of 2016, where members of the general public were invited to explore the reconstruction using virtual reality headsets and xbox controllers, and provide feedback in form of Likert-scale questionnaires and interviews. The first 
access point (a location of interest in the museum for which data was gathered from the equivalent vantage point in the virtual environment) was installed in the fall of 2016 and museum visitors were invited to interact with the system during an open day event (Fig. 1). This access point was evaluated with focus groups in the spring of 2017 and the findings are discussed in Section 6 .

\section{Design and Implementation}

Design decisions were made on interaction - using fixed-view access points, content - using spherical images, and platform - using virtual reality headsets, after which the system was implemented, beginning with research, followed by 3D modelling, content extraction and system deployment.

\subsection{Reconstruction Process}

The reconstruction process began with gathering visual evidence and culminated with the deployment of stereo equirectangular images which were extracted from an Unreal Engine 3D model.

Historical Research: Historical research entailed gathering scale plans of the building and historical images of the local community. The scale plans represented the footprint of the curing yard which has undergone minimal changes in the lifespan of the building. In addition, a map of the village Helmsdale was obtained and this served as the basis for the development of a building plan.

3D Modelling: The modelling process began in Sketchup [18] using the plan and footprint uncovered by the research phase, to ensure that the building was modelled to scale. The Sketchup 3D model (early stages shown in Fig 3), which represented the frame of the building, was then exported as a Collada mesh and imported into Unreal Engine. The model was then populated with objects that would have been found in a $19^{\text {th }}$ curing yard (as uncovered by the research), and textured with appropriate patterns to improve the photo-realism. The output of this process was a photo-realistic 3D model of the curing yard as it stood around 1890. This model - which featured troughs, barrels and buildings, as well as the surrounding landscape and river (see Fig 7) - served as the basis for content extraction and deployment.

Content Extraction: Stereo equirectangular images, formatted in a top-bottom manner (see Fig 4) were extracted from the 3D model using Unreal Engine's "Stereo Panoramic Movie Capture" plugin. This plugin provides options to change input parameters so as to control the quality of the resulting captures. These parameters include the horizontal (and vertical) angular increment (i.e. the number of slices captured horizontally and vertically as factors of $360^{\circ}$ and $180^{\circ}$ respectively), horizontal field of view (i.e. how much of the spherical environment can be viewed at any one time), and eye separation (i.e. the distance between the stereo cameras, to mirror the Interpupilary Distance [IPD]).

Deployment: The equirectangular images extracted from the model were used to make photosphere tours of the different vantage points around the curing yard. The photosphere tours were deployed as a mobile app (on Android, enabled using the OpenGL environment) which can be viewed using a mobile VR headset (such as the Google Cardboard [19]) in stereoscopic mode and without a 


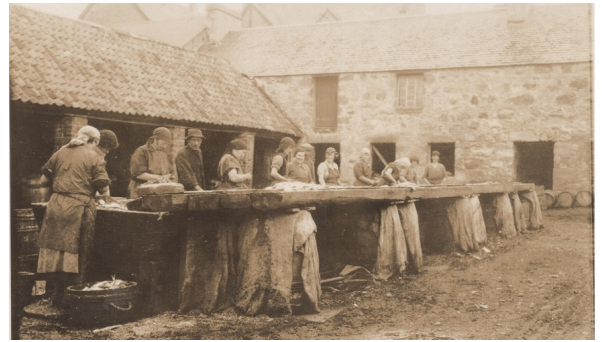

Fig. 5: Workers in a $19^{\text {th }}$-cent. curing yard

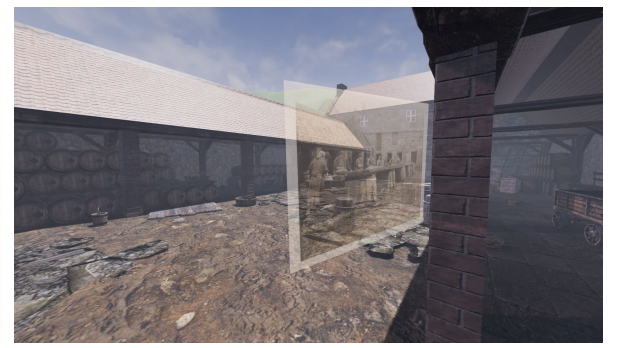

Fig. 6: View of the virtual curing yard

VR headset in wide mode, and as a web-based photosphere tour for both online use (as deployed on Roundme [20]) and offline use (enabled using krpano framework 21]) which can be navigated using an Xbox controller or with a standard keyboard and mouse. The considerations that influenced these modes of deployment are discussed in Sections 5.2 and 5.3. Historical images (see Fig 5) and text snippets were embedded into the virtual environment to provide context, draw attention to the equivalence of the real and virtual vantage points, and provide heritage interpretation. This was achieved by blending the flat, historical image onto an equirectangular, virtual image such that the historical image appeared to be superimposed on the spherical environment represented by the virtual image (see Fig 6).

\subsection{Design Decisions}

Content: A fully-immersive environment was adopted to satisfy the requirements for an engaging on-site installation. This could have been achieved by either deploying the full 3D model or deploying photospheres of vantage points. The use of the 3D model allows users to explore the 3D model of the curing yard in first-person view while photospheres restrict users to exploring the system from distinct vantage points. On the other hand, photospheres enable the deployment of spherical environments with minimal resources on a low-end workstation or smartphone, while a 3D model requires high-end computing power and graphical resources for optimal performance. In addition to the high level of engagement, an interaction-free design for exploring fixed-view points was required, hence the photosphere approach was adopted over the model approach. The restriction of users to spherical, view points also resulted in an accurate mapping of the past (virtual world view) to the present (real world view).

Hardware Platform: Mobile (smartphone with an enclosing virtual reality headset) and Desktop (PC with a tethered virtual reality headset) platforms were considered for the hardware. Although the mobile platform had relatively lower space (real estate) requirements, the Desktop platform was chosen owing to its increased computing power and lower head-tracking latency which results in a higher fidelity experience. This also gives the installation a more permanent feel than could be obtained from the use of mobile phones and headsets. The Oculus Rift 22 and the HTC Vive 23 virtual reality headsets were considered for deployment because they represent the newest generation of high-fidelity, consumer-grade headsets. The Oculus Rift was chosen as it has smaller physical space requirements as compared to the HTC Vive. 


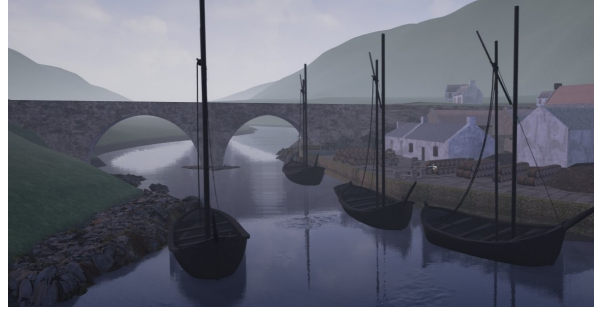

Fig. 7: Bridge \& river in Virtual Hemlsdale

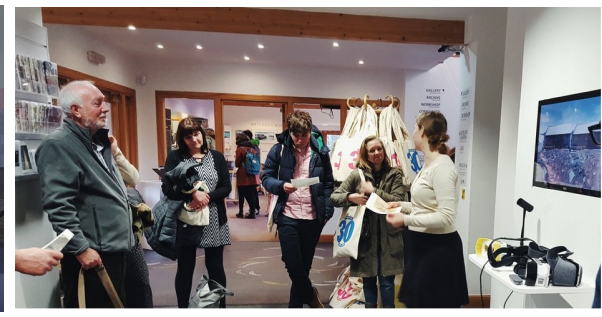

Fig. 8: Exhibit installed in the foyer

\subsection{Exhibit Installation}

As discussed in Section 5.2 a PC with a tethered virtual reality headset was chosen for the installation. The spherical environment was designed as a web-based virtual tour using krpano 21, which retrieves content from a web server running on the PC. To provide input feed into the Oculus Rift headset from the virtual tour, WebVR - an experimental JavaScript API that facilitates consumption of web-based content via virtual reality headsets - was used [24]. At the time of developing the installation, the two WebVR-compatible browsers for desktop were Chromium (an open source version of Google Chrome) and Nightly (a version of Mozilla Firefox). Chromium was chosen (over Nightly) for the installation because it exhibited greater compatibility with the system features when both browsers were tested.

A false wall was built in the museum to accommodate the computer, with a slot for the screen to fit into, outlets for cables and a protruded base for the headset to sit on as shown in Fig. 8. A decision was made to keep the installation interaction-free and easy-to-use. For this reason, interaction was limited to exploring the immediate surroundings of the virtual environment that correspond with each access point. In addition, the exhibit space was stripped of extraneous devices that did not contribute towards exploring the point of interest, thus there were no traditional computer peripherals (keyboard, mouse) or virtual reality controllers (Xbox controller, Oculus touch). This contributed towards the usability of the system, such that the installation sent a clear message to visitors: "put on the headset and look around".

Alongside the ease-of-use requirement was the ease-of-management requirement, i.e. the system should run seamlessly and require little or no input from the museum staff. This includes turning on and shutting down automatically at the start and close of business respectively. Automatic startup was achieved by changing the system BIOS setting, and automatic shutdown was achieved using the Task Scheduler feature of the Windows 7 Operating System. In addition to automatically starting up the computer, the spherical environment was automatically launched shortly afterwards. This was done through the use of a batch script that contained the commands to be executed in sequence. A link to this script was placed in the "Startup" programs folder of the computer so that it is launched after the computer boots up.

A challenge was encountered when attempting to launch the virtual reality component of the installation automatically. This resulted from an inherent security feature of web browsers which require user input (such as a mouse click or key press) in order to activate the fullscreen mode, and since the WebVR re- 


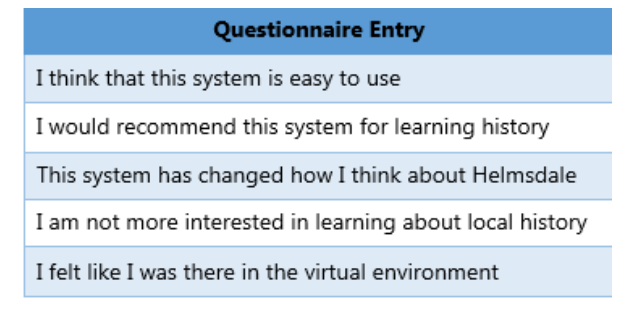

Table 1: Questionnaire items

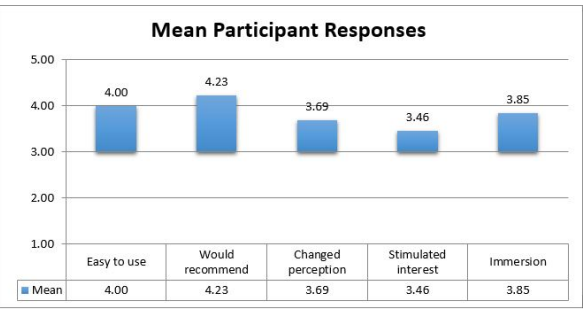

Fig. 9: Mean responses to items

quires the environment to be in fullscreen mode, any attempts to automatically launch it failed due to a lack of user input. The solution to this problem was to simulate a mouse click at the centre of the screen using a Java program, and to attach an "onclick" event to the web environment, whose action is to launch the virtual reality component. The "Startup" batch script was then modified to launch the "clicker" program shortly after launching the web-based spherical environment, thus user interaction was successfully simulated.

\section{System Evaluation}

The system has been evaluated with emphasis on usability from a user's perspective and value from a heritage expert's perspective. Usability is defined in terms of user engagement with the technology; this is investigated using Likert-scale questionnaires, semi-structured interviews and focus groups. Value is defined in terms of the contributions that the system makes towards actualising the heritage organisation's goals as perceived by the heritage experts.

A user study was conducted at the Helmsdale Highland Games that took place on the $20^{\text {th }}$ of August 2016, where attendees were invited to trial the systems. Feedback was gathered from thirteen (13) participants and the feedback was positive overall. Participants found the system very engaging and interesting, and expressed that it gives viewers an insight into the past through a captivating visual experience. The ability to explore the virtual environment at one's pace, the ability to focus on areas of interest and the superimposition of archival images on virtual content were cited as positives. One participant expressed the desire to dynamically explore the virtual environment and another mentioned a mild feeling of dizziness while using the headset. Overall, the qualitative feedback suggest that the system is suited for exploring the past in an engaging manner.

Participants also filled in a custom 5-point Likert scale (where 1 represents "strongly disagree" and 5 represents "strongly agree"). The custom questionnaire (shown in Table 1) was used instead of a standardised industry questionnaire to ensure a quick turnaround time and to directly elicit quantitative feedback on five aspects of the system: ease of use, recommendation potential, ability to change perception, ability to stimulate interest and level of immersion. The results (summarised in Fig. 99) show that participant responses were all positive (i.e. well above the neutral score of 3), hence participants found the system easy to use, participants would recommend the system for learning history, participants' perceptions of Helmsdale were changed by the system, participants became more interested in learning about local history and participants felt immersed in the virtual environment. 
In addition to the user evaluation, an expert evaluation - in the form of preand post-deployment interviews - was conducted with heritage experts at the Timespan museum and art centre. This took the form of interviews conducted before the system was deployed so as to gauge expectations, and interviews conducted after deployment to review outcomes and elicit feedback on level of satisfaction. An interview with Anna Vermehren, the Director of Timespan revealed that the museum hoped to offer novel, interactive experiences to visitors, through an installation that is informative, easy-to-use, and leaves a long-lasting impression. When asked about her views on the project output, she said:

"...the final product is definitely beyond the expectations that I've had in the beginning both visually and also in terms of the research...I really love the integration of archival material (images) because it demonstrates the research conducted".

An interview was also conducted with Dr Jo Clemens, Timespan's Archive Development Manager and the project lead. When asked about how the installation will add value to visitors, she stated that the system offers a unique experience to visitors by bridging time and space barriers, in the sense that it enables to visitors compare their current location (i.e where they are currently standing and using the system) to what it was like in the past, thus fostering a comparison of past and present and enabling them to see how it has evolved. The context of location (i.e. comparing the past and present from an equivalent vantage point) is important in achieving this, as context turns the virtual environment into something that is more than just interesting images, thus making the experience more meaningful. In addition to educating visitors, the installation will pique visitors' interests in local history and direct them to the museum archive where they can learn more.

The curing yard was evaluated with a focus group consisting of nine (9) persons (six female) at the 2017 Timespan Conference. The evaluation took the form of an open-ended discussions which lasted for approximately one (1) hour, in which participants gathered around the curing yard installation and took turns standing in front of the screen and using the virtual reality headset. A discussion ensued during which an observer took notes of the comments and participant behaviour. The notes are categorised based on the aspects of usability, content and technology as discussed below.

Usability: A discussion ensued about the ease of use of the system, as one participant remarked about how "straight-forward" the installation was. A participant stated that the position of the installation (in the museum foyer next to the Front of House desk) worked really well, as it made it easily noticeable to new museum visitors and it made a clear statement to visitors to simply "walk up and use" the system. Furthermore, the lack of interaction was cited as particularly beneficial to older-aged visitors, as users simply had to put on a headset and look around. Participants all agreed that the absence of peripherals (besides the headset and the screen) made the installation less intimidating and more inviting to users.

Content: Participants expressed curiosity about the single-view nature of installation, as one participant asked why there were not multiple locations that could be accessed in the virtual environment. A museum staff who was part 
of the discussion mentioned that the installation serves as the first in a series of three access points around the museum, where each access point provides a single, $360^{\circ}$ view of what the $19^{\text {th }}$-century curing yard would have looked like at that vantage point. This explanation seemed to "sit well" with participants. However, a participant stated that this decision made the resulting installation seem so "basic", suggesting that a more dynamic environment could have been deployed with the technology used for the installation. Also on the aspect of content, participants seemed to appreciate the blending of an archival image into the virtual environment, together with informative text snippets which fade in and out when an area of interest is looked at. Participants also suggested the addition of audio to provide more information and context about the $19^{\text {th }}$ century curing yard. One participant suggested including ambient sound in form of historical songs that were often sung by herring girls while gutting fish, and another suggested the use of audio narratives instead of (or at least together with) the text snippets for the benefit of children (who may be unable to read) and adults (who may be unwilling to browse around for the snippets).

Technology: As participants explored the curing yard, a participant asked why an Augmented Reality (AR) approach - as opposed to a Virtual Reality (VR) approach - had not been adopted for the installation. The participant suggested that a system where visitors could hold up a device in a direction in the museum foyer and be presented with a synthetic environment with informative text, may have worked better than the current installation. Another participant chimed in to state that such a system would require the use of mobile devices, which would either require purchasing multiple devices or requiring museum visitors to have capable devices with the application downloaded and installed. This would be less practical than the current installation as suggested by the participant. Furthermore, yet another participant made an argument for the use of VR instead of AR by stating that the use of VR instead of AR enables users to immerse themselves in the virtual environment (which represents the past) and compare the experience with the real environment (which represents the same space in present day). Such a comparison of experiences would be lost if a mixed (augmented) reality approach was adopted.

An evaluation also was conducted with a focus group consisting of 3 (female) participants, aged 50-70, who are all members of the Timespan Heritage Group. This exercise was particularly valuable because the participants were all experienced heritage practitioners who represent the target demographic, hence they were able to provide valuable insights. Overall, the participants stated that they were satisfied with the experience. Participants were pleased with the level of detail in the Curing yard, and they consider the system as a very informative resource and a good way to learn about heritage. The findings of the exercise are discussed in terms of user experience, technology and content.

User Experience: When asked about the user experience, a participant stated that they were pleased. One participant stated that they found the experience "very smooth" and another commented "you're really there, wow, all round you". A participant stated that "the use of old pictures blended into the virtual environment brings the exhibit to life". Conversely, another participant was displeased with the embedded historical image because "it comes in and obscures what is 


\begin{tabular}{|l||l|l|}
\hline System & Cost & User Experience \\
\hline \hline Mobile VR (Photospheres) & Low & Medium \\
\hline Desktop VR (Photospheres) & Medium & High \\
\hline Desktop VR (3D Model) & High & High \\
\hline
\end{tabular}

Table 2: Comparison of developed mobile and desktop VR systems

behind it (i.e. the virtual environment) too quickly". A participant suggested that a chair might be useful so that when users wish to make a full $360^{\circ}$ turn, it can be done safely while seated as opposed to while standing. This might mitigate the risk of falling or getting entangled by the headset wires when turning. At this point, another participant suggested that wireless headsets may improve the user experience.

Technology and Content: Participants expressed preference for the use of engaging, virtual reality technology as a means of interpretation over storytelling. In addition, participants thought that the use of virtual reality made the exhibit more exciting, and makes them excited to try new means of heritage interpretation. In terms of content, one participant suggested that the use of audio narratives would be beneficial, while another expressed preference for ambient sound (in form of voices in the background) instead of a narrative. Overall, participants expressed acceptance of the proposed addition of audio to the exhibit.

\subsection{Challenges and Limitations}

A major challenge was in determining how to maximise user experience with minimum cost. As discussed in Section 5.2, to create an immersive, spherical environment, a 3D model approach was pitted against a photosphere approach and the latter was adopted due to its relatively-lower resource requirements. A decision was then made on how best to deploy the spherical environment, at which point mobile-based virtual reality systems were pitted against desktopbased virtual reality systems. An immersive, desktop-based approach was chosen as it represented the ideal trade-offs between cost and user experience, i.e. it was found to deliver the best experience per price unit as compared to a mobile-based, photosphere approach or a desktop-based, 3D model approach. Table 2 shows the trade-offs between cost and user experience for the system configurations considered.

Another challenge manifested in form of a trade-off between ease of use (from the perspective of the museum visitors) and convenience of management (from the perspective of the museum staff). For convenience, the exhibit should be relatively easy to turn on/off and debug when issues arise, and for ease of use, the system should be relatively straight-forward to use with minimal (or no) instructions. To this end, the system was configured to automatically boot-up and shut-down on a schedule to minimise the burden on museum staff, and the interaction was made as interaction-free as possible so that visitors know how to engage with the system without being told what to do. 


\subsection{Future Work}

The next stage of the project involves deploying the model in more access points around the museum so that visitors can explore the curing yard from multiple vantage points. The new access points as well as the existing one will incorporate curing yard related sound tracks as well as audio narratives to improve on the immersive experience, and further evaluation will be conducted to evaluate whether the site-specific nature of the installation becomes more obvious to users once there are more access points, evaluate whether the target demographic (over 65s) will interact with the technology, and the extent to which the novelty of the technology contributes to the interaction, and evaluate how site-specific, virtual reality exhibits which are distributed across a museum can function as an educative tool.

\section{Conclusion}

The motivation, methodology and implementation of a $19^{\text {th }}$ curing yard virtual reconstruction have been discussed in this work. The curing yard has been deployed as an on-site museum installation that visitors can interact with to explore local heritage and compare the past and present from equivalent vantage points. The content of the museum installation has also been deployed as a mobile app for Android smartphones and as a virtual tour which is accessible via a web browser. Emphasis has been placed on an interaction-free design which delivers "snackable" content to ensure that the system easy to use, the experience is informative and engaging, and the resources required are kept to a minimum. The system evaluation has revealed positive feedback, as users at a community event who interacted with the system prototype expressed views on the value of the system in providing a means to visualise local history. Evaluation was also conducted with heritage experts to investigate the potential value of the system from the perspective of heritage organisations. The feedback was positive, with experts stating how the mix between archival images and generated computer graphics brings the past to life. In addition to these, focus group evaluations have revealed that the system is ease of use, features informative content and offers an engaging experience for group exploration. These findings suggest that museums and heritage organisations can provide compelling and informative experiences that enable visitors to travel back in time with minimal interaction and relatively low cost systems.

\section{References}

1. Bruno, F., Bruno, S., De Sensi, G., Luchi, M. L., Mancuso, S., \& Muzzupappa, M. 2010. From 3D reconstruction to virtual reality: A complete methodology for digital archaeological exhibition. Journal of Cultural Heritage, 11(1), 4249. http://doi.org/10.1016/j.culher.2009.02.006

2. Styliani, S., Fotis, L., Kostas, K., \& Petros, P. (2009). Virtual museums, a survey and some issues for consideration. Journal of Cultural Heritage, 10, 520528. http://doi.org/10.1016/j.culher.2009.03.003

3. Woodwark, J. 1991. Reconstructing history with computer graphics. IEEE Computer Graphics and Applications, 11(1), 18-20. 
4. Grau, O. 2003. Virtual Art: from illusion to immersion. MIT press. Chicago

5. Cameron, F., \& Kenderdine, S. "Speaking in Rama: Panoramic Vision in Cultural Heritage Visualization" in Theorizing Digital Cultural Heritage: A Critical Discourse, Cambridge, MA, England: MIT Press, 2007, ch. 15, pp.301-331.

6. McCaffery, J., Miller, A., Vermehren, A., \& Fabola, A. (2015, September). The Virtual Museums of Caen: A case study on modes of representation of digital historical content. In 2015 Digital Heritage (Vol. 2, pp. 541-548). IEEE.

7. Kennedy, S., Fawcett, R., Miller, A., Dow, L., Sweetman, R., Field, A., Campbell, A., Oliver, I., McCaffery, J., and Allison, C. "Exploring canons \& cathedrals with Open Virtual Worlds: The recreation of St Andrews Cathedral, St Andrews day, 1318" 2013 Digit. Herit. Int. Congr., pp. 273-280, Oct. 2013.

8. Kiourt, C., Koutsoudis, A., \& Pavlidis, G. (2016). DynaMus: a fully dynamic 3D virtual Museum framework. Journal of Cultural Heritage, 22, 984-991.

9. Rodrguez, M. B., Agus, M., Bettio, F., Marton, F., \& Gobbetti, E. (2015, September). Digital Mont'e Prama: 3D Cultural Heritage presentations in museums and anywhere. In 2015 Digital Heritage (Vol. 2, pp. 557-564). IEEE.

10. Kostadinov, S. D., \& Vassilev, T. I. (2013). MOBILE VIRTUAL 3D MODEL OF A MEDIEVAL TOWN. International Journal on Information Technologies \& Security, 5(4), 13-18.

11. Tait, E., Laing, R., Grinnall, A., Burnett, S., \& Isaacs, J. (2016). (Re) presenting heritage: Laser scanning and $3 \mathrm{D}$ visualisations for cultural resilience and community engagement. Journal of Information Science, 42(3), 420-433.

12. Galena Kostoska, Marcos Baez, Florian Daniel, and Fabio Casati. Virtual, remote participation in museum visits by older adults: a feasibility study. In Proceedings of the 8th International Conference on Personalized Access to Cultural HeritageVolume 1352, pages 10-13. CEUR-WS. org, 2015.

13. Frisoli, A., Jansson, G., Bergamasco, M., \& Loscos, C. (2005, March). Evaluation of the pure-form haptic displays used for exploration of works of art at museums. In World haptics conference, Pisa, March (pp. 18-20).

14. Carrozzino, M., Evangelista, C., Scucces, A., Tecchia, F., Tennirelli, G., \& Bergamasco, M. (2008, September). The virtual museum of sculpture. In Proceedings of the 3rd international conference on Digital Interactive Media in Entertainment and Arts (pp. 100-106). ACM. Chicago

15. Loch, J. (1820). An Account of the Improvements on the Estates of the Marquess of Stafford: In the Counties of Stafford an Salop, and on the Estate of Sutherland. With Remarks. Longman, Hurst, Rees, Orme \& Brown.

16. FSBI Publications [Online]. Available: http://www.fsbi.org.uk/publications/ council-reports/ [Accessed: 10 October 2016].

17. Unreal Engine [Online]. Available: https://www. unrealengine.com/, [Accessed: 10 October 2016].

18. Sketchup [Online]. Available: http://www.sketchup.com/ [Accessed: 10 October 2016].

19. Google. Google Cardboard [Online]. Available: https://www.google.com/get/ cardboard/ [Accessed: 10 May 2016].

20. Roundme [Online]. Available: https://roundme.com/ [Accessed: 10 May 2016].

21. krpano. krpano Panorama Viewer [Online]. Available: https://krpano.com/ [Accessed: 10 May 2016].

22. Oculus. rift + touch [Online]. Available: https://www3.oculus.com/en-us/rift/. [Accessed: 10 May 2016].

23. HTC. Vive [Online]. Available: https://www.vive.com/uk/ [Accessed: 10 May 2016].

24. WebVR. Bringing Virtual Reality to the Web [Online]. Available: https://webvr. info/. [Accessed: 10 May 2016]. 